\title{
Prácticas docentes y desarrollo de conducta prosocial en alumnos de primer ciclo básico de una escuela chilena
}

\section{Teaching practices and development of prosocial behavior in sudents of first basic cycle of a Chilean school}

\author{
María-Gracia González Navarro ${ }^{1}$, \\ Gracia Navarro Saldaña ${ }^{2}$
}

\begin{abstract}
RESUMEN
Se identifican las prácticas docentes de profesores de un establecimiento educacional chileno, con alto índice de vulnerabilidad social (90.5\%), y su relación con la aparición de conductas prosociales. Se analizó una muestra de siete profesores jefes del primer ciclo básico, a través de entrevista y observación directa. Los resultados muestran que el Comportamiento Normativo de los profesores tiene impacto en la aparición de conductas prosociales en los alumnos y que éstas se ven obstruidas por la inconsistencia en las normas y por el énfasis en la figura de autoridad para su cumplimiento. Las prácticas docentes no están promoviendo el desarrollo moral de los alumnos y, por lo tanto, no facilitan la aparición de conductas prosociales, las cuales funcionan como factor protector ante la conducta violenta.
\end{abstract}

Así, la figura del profesor es fundamental para el desarrollo de conductas prosociales, siendo efectivas en cuanto generan o no oportunidades para el desarrollo de la moralidad.

Palabras claves: Práctica Docente - Conducta Prosocial - Normas - Convivencia Escolar

\footnotetext{
${ }^{1}$ Universidad de Concepción. Chile. Teléfono: +56412204996. Correo electrónico: mariagraciagn@gmail.com ${ }^{2}$ Universidad de Concepción. Chile. Teléfono: +56412204996. Correo electrónico: gnavarro@udec.cl
}

Recepción: 08/10/2019 Aceptación: 20/01/2020 Doi: 10.15517/WL.V15I1.40483 
6 OЙв Lu Wimblu, Rev. Estud. de Psicología UCR, 15(1) 2020 (Enero-Junio): 7-34 /ISSN: 1659-2107

\begin{abstract}
This research identifies teaching practices of teachers in a Chilian school with high index of social vulnerability (90.5\%), and its relation to the development of prosocial behavior. A sample of seven teachers of basic school were analized by interviews and observation made to their classes.

The results show that the normative behavior of teachers has an impact on the development of prosocial behavior in students and that they are blocked by inconsistency in the rules and the emphasis in the authority figure for it compliance. Teaching practice is not promoting the moral development of students and therefore do not facilitate the emergence of prosocial behaviors, which function as a protective factor against violent behavior. It is concluded that the professor is critical to the development of prosocial behaviors and practices that become effective creating opportunities for the development of morality.
\end{abstract}

Key Words: Teaching practice - Prosocial behavior - Normative behavior - school climate

\title{
1. Marco Teórico
}

Para que el aprendizaje sea posible, los intercambios entre todos los actores de la institución (alumnos, docentes y padres) que comparten la actividad en la escuela y que conforman la red de vínculos interpersonales, debe construirse cotidianamente, mantenerse y renovarse según determinados valores. Así, sólo cuando en una institución escolar se privilegian la comunicación, el respeto mutuo, el diálogo y la participación, recién entonces se genera el clima adecuado para posibilitar el aprendizaje ${ }^{12}$. La educación requiere de la adquisición de conocimientos teóricos e información y de la capacidad de utilizarlos como herramientas útiles y prácticas para el desempeño de tareas cotidianas; siguiendo a Renes y Caldeiro $^{3}$, se puede sostener que es necesario un proceso educativo en el que prime tanto el aprender a conocer como el aprender a hacer, sin olvidar que la persona en tanto ser social requiere de la adquisición de una serie de destrezas personales que cobran sentido en su contexto social.

\footnotetext{
1 N. Ianni, La convivencia escolar: una tarea necesaria, posible y compleja. Monografías virtuales: Ciudadanía, democracia valores en sociedades plurales http://www.oei.es/valores2/monografias/monografia02/reflexion02.htm

2 D. Macías, A. Santamaría, A. Zavala, M. Adoví y J. Pincay, Gestión pedagógica: clima social en el aula, desde la percepción de estudiantes y profesores. Revista electrónica Formación y Calidad Educativa REFCalE 4, no. 1 (2016).

${ }^{3}$ Renes P. y Caldeiro MC. (2017) Nuevos escenarios sobre la educación en valores en las escuelas. Inovare 4, no. 2 (2017)
} 
La convivencia y el aprendizaje se condicionan mutuamente. La causalidad circular permite comprender la interrelación entre ambos: cada uno es condición necesaria (aunque no suficiente por sí solo) para que se dé el otro ${ }^{4}$. Así una convivencia escolar positiva, fundamentada en principios y valores, puede facilitar y catalizar el proceso de enseñanzaaprendizaje.

A pesar de la importancia de una adecuada convivencia escolar, en los últimos años, la violencia en las escuelas ha empezado a ser protagonista, cada vez más frecuente, de los medios de comunicación masiva ${ }^{5}$. Investigaciones postulan que aproximadamente la mitad del alumnado ha visto o ha participado en conductas violentas que tienen que ver con insultos o peleas ${ }^{6}$.

La conducta violenta se entiende como los comportamientos agresivos que llegan a causar daño físico o moral ${ }^{7}$. La naturaleza y características de la conducta violenta pueden variar, pero generalmente incluyen actos relacionados con la violencia física con las personas, violencia verbal contra las personas y la violencia o deterioro deliberado de las cosas. Algunos autores mencionan conductas o actuaciones (a veces la conducta violenta se expresa también desde la no intervención) que constituyen formas de desprecio, minusvaloración o marginación de los sujetos víctimas ${ }^{8}$.

\footnotetext{
${ }^{4}$ N. Ianni, La convivencia escolar: una tarea necesaria, posible y compleja. Monografías virtuales: Ciudadanía,

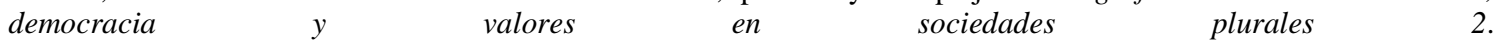
http://www.oei.es/valores2/monografias/monografia02/reflexion02.htm

${ }^{5}$ C. Mertz, La prevención de la violencia en las escuelas. Programa Paz Educa (2006).
}

\footnotetext{
${ }^{6}$ Cangas, Vázquez, Pérez-Fuentes, Padilla y Miras, Evaluación de la violencia escolar y su afectación personal en una muestra de estudiantes europeos. Psicothema 19 (2007): $114-119$.

7 M. Zabalza, Situación de la convivencia escolar en España: Políticas de intervención. Revista Interuniversitaria de Formación de Profesorado 44 (2002): 139 - 174.

${ }^{8}$ M. Zabalza, Situación de la convivencia escolar en España: Políticas de intervención. Revista Interuniversitaria de Formación de Profesorado 44 (2002): 139 - 174.
} 


\subsection{Conducta prosocial como factor protector de la conducta violenta}

Alonso, Cáceres e Hinojo ${ }^{9}$ conciben la escuela como el espacio educativo en el que la violencia puede ser prevenida. Dentro de las principales razones por las cuales la violencia en las escuelas debe ser prevenida está que dificulta el aprendizaje de los alumnos, les causa daños físicos y psicológicos a las víctimas de ella, por ejemplo la baja autoestima ${ }^{10}$. Además, está que el involucramiento en la violencia es un factor de riesgo que incrementa las probabilidades de emprender trayectorias de vida problemáticas ${ }^{11}$.

Paralelamente se ha concluido que los factores protectores de comportamientos problemáticos, violencia entre ellos, son en términos generales, la provisión de oportunidades de tener interacciones y realizar acciones prosociales, el reforzamiento positivo de las acciones prosociales, la provisión de creencias sanas, estándares de comportamientos claros y la formación de vínculos afectivos o emocionales en todos los ámbitos sociales con personas que tiene dichas creencias (familia, escuela, grupo de pares y comunidad $)^{12}$.

Conducta Prosocial: La conducta prosocial se entiende como los comportamientos llevados a cabo voluntariamente para ayudar o beneficiar a otros, tales como compartir, dar apoyo y protección ${ }^{13}$. Es, además, una de las conductas a través de las cuales se ejerce la responsabilidad social y como tal, su generación y desarrollo se relaciona directamente con el desarrollo de la moralidad ${ }^{14}$.

\footnotetext{
${ }^{9}$ S. Alonso, M. Cáceres y M. Hinojo, La Convivencia Escolar y el Conocimiento de un Grupo Mediante la Sociometría: Entrenamiento en Habilidades Sociales. Revista Iberoamericana sobre Calidad, Eficacia y Cambio en Educación 8 (2010): 45-57.

${ }^{10}$ M. De la Villa, A. García, G. Cuetos y C. Sirvent, Violencia en el noviazgo, dependencia emocional y autoestima en adolescentes y jóvenes españoles. Revista Iberoamericana de Psicología y Salud. 8, no. 2 (2017): 96-107.

${ }^{11}$ C. Mertz, La prevención de la violencia en las escuelas. Programa Paz Educa (2006).

${ }^{12}$ C. Mertz, La prevención de la violencia en las escuelas. Programa Paz Educa (2006).

${ }^{13}$ M. Martí,. Razonamiento moral y prosocialidad (Madrid: Editorial CCS, 2010).

${ }^{14}$ G. Navarro, G. Vaccarezza, M-G. González y R. Catalán, Construcción de conocimiento en educación superior; educación de competencias genéricas en la Universidad de Concepción (Concepción, Editorial Universidad de Concepción, 2015).
} 


\subsection{Desarrollo moral}

Según Fuentes ${ }^{15}$, en el ámbito de la pedagogía, la educación moral es un tema clásico y recurrente en las investigaciones, que necesita constantemente de nuevas miradas que permitan reinterpretar las mejores aproximaciones en un contexto sociohistórico cambiante donde los enfoques y el peso que se le ha concedido han variado notablemente entre las diferentes corrientes de pensamiento. No obstante, previo a la educación moral es necesario referirse al desarrollo moral para el cual las investigaciones permiten sostener, siguiendo a Fierro y Carbajal ${ }^{16}$, que el individuo pasa por tres etapas importantes en el desarrollo de su moralidad: La primera es la de socialización, en la que se transmiten valoraciones y se pretende que el sujeto se adapte a las normas vigentes en una sociedad determinada. La segunda es la de interiorización de las normas, es decir, cuando el sujeto logra hacerlas propias (tanto las que obedecen a principios éticos universales como las específicas de una sociedad determinada); cuando tiene que tomar decisiones, se inclina por respetarlas. La tercera está caracterizada por una moral autónoma, y en ella el sujeto es capaz de cuestionar la normatividad vigente en su propia cultura y se guía por principios éticos universales, elegidos de manera autónoma, cuando enfrenta alguna situación de carácter moral en la que tiene que tomar una decisión.

$\mathrm{Si}$ se considera la conducta prosocial como comportamientos voluntarios, llevados a cabo para ayudar o beneficiar a otros, tales como compartir, dar apoyo y protección ${ }^{17} 18$, para que el comportamiento prosocial perdure aún en ausencia de autoridades, un niño debe desarrollarse moralmente para tener conductas de este tipo e interiorizar el comportamiento socialmente responsable.

\footnotetext{
15 J.L. Fuentes, Evolución y perspectivas de la educación moral. Revista española de pedagogía REP 75 (2018).

${ }^{16}$ C. Fierro y P. Carbajal, El docente y los valores desde su práctica. Sinéctica 22 (2003): 3 - 11.

${ }^{17}$ M. Martí,. Razonamiento moral y prosocialidad (Madrid: Editorial CCS, 2010).

${ }^{18}$ C. Inglés, E. Martínez-González y J. García-Fernández, Conducta prosocial y estrategias de aprendizaje en una muestra de estudiantes españoles de Educación Secundaria Obligatoria European Journal of Education and Psychology. 6, no. 1 (2013): 33-53
} 


\subsection{Prácticas docentes y desarrollo moral}

Dentro de las variables que determinan la aparición de conductas prosociales están los factores del contexto de socialización y entre ellos la interacción con el profesorado ${ }^{19}$. Es este último uno de los responsables directos de la implantación de una educación basada en una ideología integral, que considere aspectos académicos y valorativos igualmente relevantes que permita el desarrollo de conductas prosociales ${ }^{20}$.

Dentro de aquella interacción, se encuentran las prácticas docentes, las cuales se entienden como las actividades cotidianas realizadas, orientada por un currículo, en un contexto escolar y social, dirigidas a la construcción de saberes y formación de los estudiantes como vía para el desarrollo personal y la convivencia social ${ }^{21}$. Por lo tanto, tienen el poder de promover o dificultar la generación de ambientes que promuevan el desarrollo de la moralidad y así, la perpetuación de los comportamientos prosociales. Sin embargo, esto no depende sólo de la enseñanza intencional del maestro, sino sobre todo de los valores experimentados en las acciones cotidianas hacia las situaciones normativas que confronta en la sala de clases, esto debido a que los alumnos generalmente están valorando, ponderando, eligiendo y poniendo en práctica sus valores ${ }^{22}$. Apoyar el desarrollo moral de los alumnos significa acompañarlos en la reflexión sobre los conflictos suscitados y en la adopción de una solución determinada, guiada por el principio de justicia ${ }^{23}$.

Dentro del proceso valorativo de los alumnos que lleva a la conformación de conductas prosociales o conductas violentas, están a) Los valores que porta el alumno respecto de lo bueno, justo o malo, b) Las actitudes valorativas expresadas en sus argumentos, en la situación normativa, c) Las acciones intencionales realizadas por el alumno y d) El saber moral práctico. A su vez, cada uno de estos aspectos se ve influido por las características de

\footnotetext{
${ }^{19}$ M. Martí,. Razonamiento moral y prosocialidad (Madrid: Editorial CCS, 2010).

${ }^{20}$ A. Pascual, Implicaciones psicopedagógicas de un desarrollo moral íntegro: La educación holística (Universidad de Zaragoza: España, 1999)

21 J. Quintero, Estrategias docentes como práctica de la teoría pedagógica. CONHISREMI, Revista Universitaria de Investigación y Diálogo Académico 4 (2008).

${ }^{22}$ M.G. Velázquez, M.G. y A. Candela, Educación, valores y desarrollo moral. Formación valoral y ciudadana, Gernika 2 (2006) 335-356.

${ }^{23}$ C. Fierro y P. Carbajal, El docente y los valores desde su práctica. Sinéctica 22 (2003): 3 - 11.
} 
la interacción alumno-profesor, y se ve muy beneficiado cuando este último posee una autenticidad psicológica (es real en la comunicación con los estudiantes) y moral (coherente entre lo que dice y sus actos, sobre todo en el desarrollo de valores). Se requiere que el maestro esté atento a las necesidades del educando desde una actitud de aceptación, confianza y cuidado con el alumno, que sea una persona comprometida con su trabajo de educador y formador de personas íntegras. Para ello él debe ser también modelo y enseñar desde la autenticidad psicológica y moral. El profesor debe incitar la motivación de los alumnos a la participación en los procesos de la educación moral, crear un aula de tolerancia entre el alumnado, promover situaciones de diálogo y debate moral, procurar actitudes de comunicación y de escucha, saber ser mediador en los conflictos, seguir el proceso de crecimiento de la persona con respeto y paciencia, y aceptar con responsabilidad y humildad el papel de experto moral ${ }^{24}$.

\subsection{Modelo oferta valoral docente}

Fierro y Carbajal $^{25}$, ofrecen un modelo que permite comprender cómo las prácticas docentes influyen en la generación de ambientes que promuevan el desarrollo de moralidad, ellas proponen el concepto de Oferta Valoral del Docente (OVD), entendiéndolo como aquello que el profesor transmite en términos de valores y las oportunidades que ofrece para el desarrollo de la moralidad de sus alumnos.

Se describen tres formas a través de las cuales los docentes generan aquellas oportunidades: El Comportamiento normativo, el Comportamiento afectivo y la Conducción de los procesos de enseñanza.

\footnotetext{
${ }^{24}$ A. Pascual, Implicaciones psicopedagógicas de un desarrollo moral íntegro: La educación holística (Universidad de Zaragoza: España, 1999)

${ }^{25}$ C. Fierro y P. Carbajal, El docente y los valores desde su práctica. Sinéctica 22 (2003): 3 - 11.
} 
6 OИлв Lu Wimblu, Rev. Estud. de Psicología UCR, 15(1) 2020 (Enero-Junio): 7-34 /ISSN: 1659-2107

\subsubsection{Comportamiento Normativo}

Es el conjunto de parámetros que el profesor establece para regular la conducta de los alumnos en la sala de clases y en la escuela, cobrando importancia el tipo de norma utilizada y la consistencia en el comportamiento del docente ${ }^{26}$.

$\mathrm{Al}$ considerar las normas como expresión de valores, el comportamiento del docente cobra relevancia, ya que representa la declaración de los valores que ofrece a sus alumnos en lo cotidiano. Por lo tanto, a través del comportamiento que el profesor considera obligatorio o deseable establece aspectos normativos en la sala de clases, filtrando en distintos planos valores sociales, orientaciones explicitas o implícitas de la institución escolar y sus propios valores personales.

Este comportamiento tiene relación con las normas concretas a las cuáles hace alusión el profesor durante la clase. Estas se entienden como el conjunto de prescripciones de carácter obligatorio y general, cuya transgresión conlleva consecuencias de distinto tipo, que abarcar desde hacer notar el incumplimiento hasta la sanción propiamente. Se expresan principalmente a través de lo que declara el docente y el reglamento escrito. Siendo las alusiones verbales la principal forma. Éstas pueden ser de dos tipos: Llamadas de atención $\mathrm{o}$ indicaciones.

El comportamiento normativo considera también las normas abstractas, expresadas principalmente a través de juicios de valor, entendidos como pronunciamientos sobre situaciones deseables o no deseables, comportamientos preferibles a otros o sobre la bondad o maldad en determinadas acciones.

Las normas concretas están referidas principalmente al sistema de usos y costumbres, mientras que las abstractas se refieren a los llamados "valores universales".

Por último, el comportamiento normativo se refiere también a la consistencia con la cual el profesor hace cumplir las normas. La consistencia será entendida como el grado en que las normas son aplicadas de manera regular y previsible, teniendo grandes repercusiones en la generación de oportunidades para que los alumnos las interioricen o no. Por el contrario, al ser flexibilizadas aumentan el grado de dependencia de la actividad de la figura de autoridad para su cumplimiento.

\footnotetext{
${ }^{26}$ C. Fierro y P. Carbajal, El docente y los valores desde su práctica. Sinéctica 22 (2003): 3 - 11.
} 


\subsubsection{Comportamiento Afectivo}

Se entiende a partir del vehículo utilizado por el profesor y las expresiones afectivas dirigidas a los alumnos.

Vehículo: es el medio a través del cual el maestro presenta o señala una norma concreta o abstracta. Está descrito según el grado de referencia a la norma y el uso de la autoridad para cumplirla. Representa oportunidades de toma de rol, al generar espacios para ponerse en el lugar de otros, ser conscientes de sus pensamientos y sentimientos, se favorece la interiorización de las normas y, en consecuencia, el desarrollo de la autonomía ${ }^{27}$.

Los docentes pueden usar uno o varios vehículos para hacer cumplir las normas. Los vehículos utilizados representan en sí mismos vivencias en las cuales se construyen o no los valores de justicia y respeto. Al basarse en el uso de explicaciones, se explicitan las razones y los motivos por los cuales una norma es importante, teniendo en cuenta los derechos y necesidades de los demás. Al mismo tiempo afirman con acciones que el alumno merece un trato considerado, comunicándole si el respeto a su persona está por encima o no de cualquier corrección que el profesor haga sobre su comportamiento. Cada vez que el vehículo usado no es proporcional o no es adecuado, los alumnos tienden a centrarse más en restaurar el daño recibido, quedando fuera del foco el mensaje sobre el cual el docente intenta reflexionar. Esto corrobora la importancia de utilizar vehículos adecuados cuando el objetivo es la interiorización y autonomía moral de los alumnos ${ }^{28}$.

Expresiones afectivas: Se definen como gestos de atención y consideración hacia demandas, necesidades, intereses o problemas de los alumnos; su importancia radica en la experiencia de recibir o no un trato equitativo en la escuela. Otorgan una tonalidad específica a las interacciones cotidianas entre docente y alumnos, por lo tanto, explican las diferencias en el clima percibido en diferentes salas de clases. Las expresiones afectivas no son neutras; al otorgar un trato que podría colocar al alumno en desventaja con respecto a

\footnotetext{
${ }^{27}$ C. Fierro y P. Carbajal, El docente y los valores desde su práctica. Sinéctica 22 (2003): 3 - 11.

${ }^{28}$ C. Fierro y P. Carbajal, El docente y los valores desde su práctica. Sinéctica 22 (2003): 3 - 11.
} 
los demás contribuyen a construir la vivencia de un trato arbitrario y desigual como experiencia cotidiana en la escuela.

Las expresiones afectivas se mueven en un continuo que va desde la mayor a la menor atención (o deferencia del profesor hacia el alumno) y el ejercicio de la autoridad.

\subsubsection{Conducción de los procesos de enseñanza}

Los procesos de enseñanza pueden ser conducidos a través de actividades de enseñanza expositivas o mecánicas, o actividades que promueven secuencias de reflexión.

Una secuencia de reflexión corresponde a actividades a través de las cuales el docente promueve en el alumno la forma de juicio sobre diversos comportamientos. Se caracteriza por incorporar información y juicios de valor emitidos por el docente y tiene diferentes niveles de elaboración, que pueden ir desde la generación de una o dos preguntas que motivan la reflexión, hasta una secuencia que implica un nivel superior de análisis y participación de los alumnos.

La importancia de la reflexión radica en que cuando los alumnos tienen la posibilidad de analizar situaciones de la vida diaria o dilemas morales a partir de cuestionamientos sobre ellas, elaboran sus propios razonamientos y los juicios de valor que emiten los docentes tienen la posibilidad de dialogar con los puntos de vista de los alumnos ${ }^{29}$. Todo ello representa oportunidades para que los alumnos avancen en el desarrollo de su moralidad.

Fierro y Carbajal ${ }^{30}$ definen dos tipos de reflexión, en primer lugar está la reflexión académica, en la cual el docente invita al alumno a observar, experimentar y sacar conclusiones. Promueven el desarrollo del pensamiento lógico formal a través de la participación de los alumnos en actividades académicas diversas que demandan procesos cognitivos complejos. Sin embargo, a partir de lo indicado por Kohlberg ${ }^{31}$, aunque la reflexión académica es una condición necesaria para el desarrollo del juicio moral, éste requiere del desarrollo de la perspectiva social a través de la toma de rol, es decir, ponerse en lugar del otro, saber que todas las acciones tienen consecuencias y pueden afectar a los

\footnotetext{
29 J. Ruiz, A. Ponce de León, E. Sanz y M. Valdemoros, La educación en valores desde el deporte: investigación sobre la aplicación de un programa integral en deportes de equipo RETOS. Nuevas Tendencias en Educación Física, Deporte y Recreación, no. 28 (2015): 270-275

${ }^{30}$ C. Fierro y P. Carbajal. Mirar la práctica docente desde los valores (Mexico: Gedisa, 2003)

${ }^{31}$ C. Fierro y P. Carbajal. Mirar la práctica docente desde los valores (Mexico: Gedisa, 2003)
} 
demás. Esto implica rescatar tanto los aspectos cognitivos como los afectivos de las interacciones sociales. En segundo lugar, está la reflexión valoral en la cual, a partir de un contenido académico, se invita al alumno a reflexionar sobre las consecuencias de ciertas acciones en relación con otras. Las secuencias de reflexión valoral ofrecen la oportunidad de ampliar la perspectiva social, pues cuando se puede entender lo que siente el otro en relación con ciertos acontecimientos, se generan oportunidades de pasar de la perspectiva egocéntrica a la sociocéntrica. Asimismo, hay oportunidades de interiorizar las normas y, por lo tanto, se puede generar en la persona un conflicto moral.

Tipo de Reflexión Valoral: Se describe de acuerdo a los ejes de análisis: sentido de la norma y uso de la autoridad (componentes centrales en la generación de oportunidades para interiorizar las normas) ${ }^{32}$.

En la experiencia de los autores ${ }^{33}$, cada vez que se identificaron secuencias de reflexión de contenido académico o valoral en un salón de clases los incumplimientos de normas concretas prácticamente desaparecieron, de lo cual los autores concluyen que si se propusiera a los alumnos actividades que los reten a reflexionar, se evitaría la reiteración excesiva en las normas concretas. En lugar de insistir en la normatividad se insistiría en las secuencias de reflexión, las cuales pueden plantearles una manera distinta de trabajar que repercuta directamente en su comportamiento y disminuya las transgresiones a las normas $^{34}$.

\footnotetext{
${ }^{32}$ C. Fierro y P. Carbajal, El docente y los valores desde su práctica. Sinéctica 22 (2003): 3 - 11.

${ }^{33}$ C. Fierro y P. Carbajal, El docente y los valores desde su práctica. Sinéctica 22 (2003): 3 - 11.

${ }^{34}$ C. Fierro y P. Carbajal, El docente y los valores desde su práctica. Sinéctica 22 (2003): 3 - 11.
} 
Figura 1

Modelo oferta valoral docente, propuesto por Fierro y Carbajal ${ }^{35}$

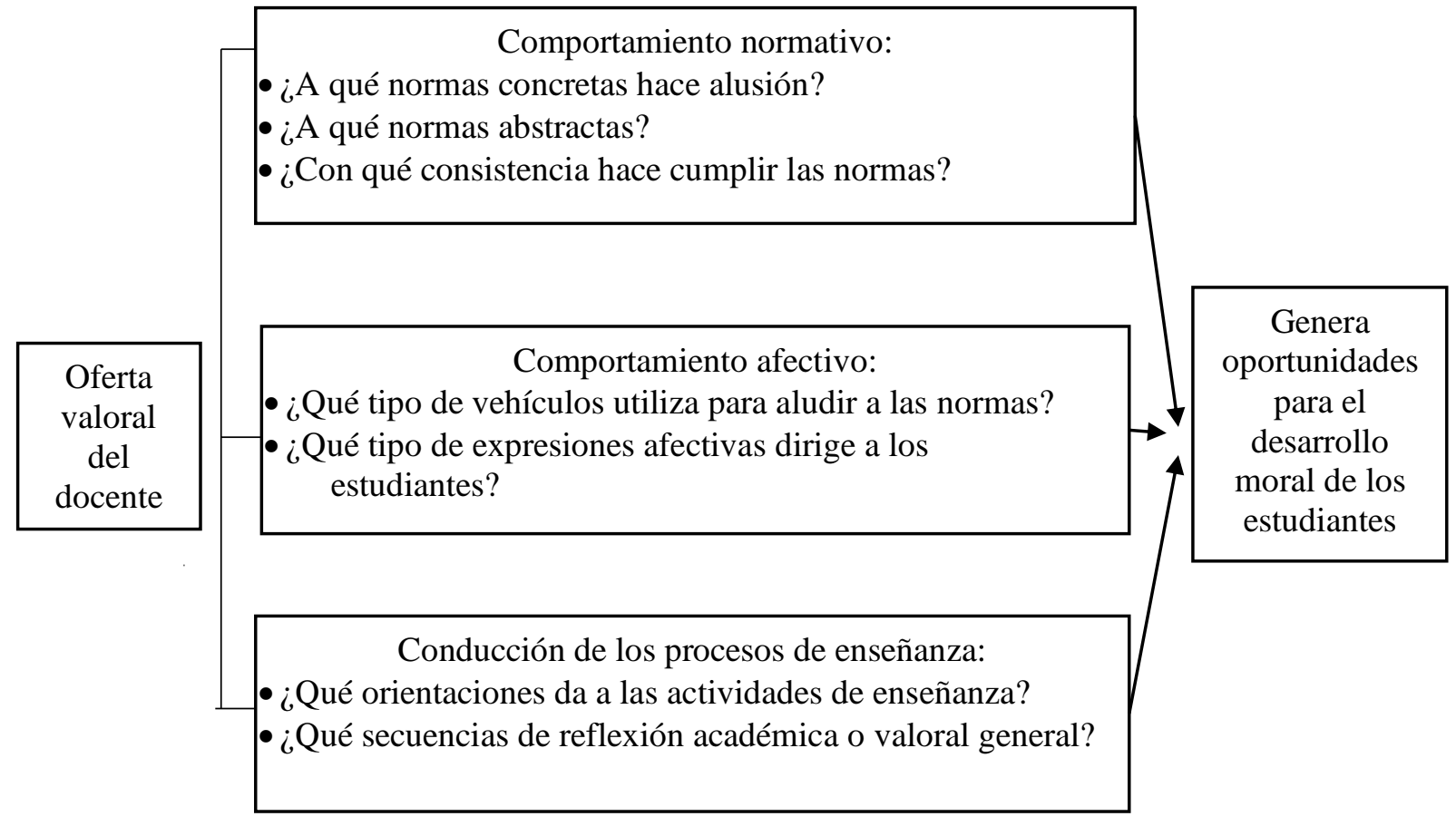

La Escuela en la que se lleva a cabo este estudio, está ubicada en la comuna de San Pedro de la Paz de Chile y atiende una población escolar que al momento de este estudio tiene serias carencias o falta de medios para uso del tiempo libre y un alto índice de Vulnerabilidad social (90.5\%) que afecta los procesos de aprendizaje y enseñanza que se viven diariamente. El índice de vulnerabilidad social (IVS) es una medida compuesta por cinco dimensiones de los riesgos o vulnerabilidad de la población chilena: el analfabetismo de la población adulta, la desnutrición en los niños/as, la pobreza de consumo en los hogares, el riesgo de mortalidad de los niños/as menores de un año, y la presencia de comunidades étnicas rurales. Se presenta en una escala de 0 a 100 donde un mayor valor representa mayor vulnerabilidad social.

Del diagnóstico psicoeducativo realizado en el mismo establecimiento, se desprende que uno de los principales problemas detectados por la comunidad educativa es la conducta violenta, la cual ha ido en aumento durante los últimos años. Frente a esto, los docentes del

${ }^{35}$ C. Fierro y P. Carbajal, El docente y los valores desde su práctica. Sinéctica 22 (2003): 3 - 11. 
establecimiento indican que los recursos de los cuales disponen para enfrentar el problema son muy reducidos. Por estar inserta en un contexto caracterizado por alta deprivación social, deficiente acceso a patrones culturales, alto nivel de pobreza, drogadicción, delincuencia, alcoholismo y violencia intrafamiliar, el ambiente que los rodea se vuelve un factor de riesgo. A partir de esto y considerando a la conducta prosocial como un factor protector frente a la conducta violenta ${ }^{36}$ surge la siguiente pregunta de investigación: ¿Existe relación entre las prácticas docentes y la aparición de conductas prosociales entre los alumnos? La hipótesis que orienta la investigación indica que las prácticas docentes favorecen o dificultan la convivencia escolar, a través de su influencia en el desarrollo de las conductas prosociales o violentas entre los estudiantes.

\section{Objetivo general}

Observar la relación entre prácticas docentes y presencia de conductas prosociales en la interacción de los alumnos del primer ciclo básico de una escuela municipal de la comuna San Pedro de la Paz, región del Bío Bío, Chile.

Objetivos específicos

- Describir prácticas docentes de los profesores del primer ciclo básico

- Describir interacción entre alumnos del primer ciclo básico, desde la declaración de apoderados, docentes y directivos de la escuela.

- Identificar las prácticas docentes de los profesores que facilitan o dificultan la aparición de conductas prosociales en la interacción entre alumnos

- Relacionar las prácticas docentes detectadas con la conducta de los estudiantes, declarada por apoderados, docentes y directivos de la escuela.

\footnotetext{
${ }^{36}$ C. Mertz, La prevención de la violencia en las escuelas. Programa Paz Educa (2006).
} 
Oीлив Lu Wimblu, Rev. Estud. de Psicología UCR, 15(1) 2020 (Enero-Junio): 7-34 /ISSN: 1659-2107

\section{Método}

La presente investigación es de diseño exploratorio descriptivo y se focaliza en una población de profesores jefes de primer ciclo básico de una escuela municipal de la comuna San Pedro de la Paz, región del Bío Bío, Chile. Utiliza la observación áulica por parte de una estudiante en práctica de Psicología Educacional a los docentes, para identificar fortalezas y dificultades en sus interacciones con los estudiantes con el propósito de ayudarles en la comprensión del fenómeno de la violencia escolar y en el autoanálisis, autoevaluación y mejoramiento de sus prácticas docentes, para fortalecer la prosocialidad. Además, realiza entrevistas acerca de las interacciones de los estudiantes en diferentes espacios y observaciones de las mismas.

La muestra se constituye a partir del cumplimiento del criterio: Tener Clases de Lenguaje y Comunicación en el primer bloque de la jornada, es decir, 08:00 am. Se elige este horario para disminuir la influencia del agotamiento de los docentes y se elige la asignatura porque según el Marco Curricular de Enseñanza Básica y Media de Chile ${ }^{37}$ es la clase que puede posibilitar mayor interacción profesor-alumnos en torno a contenidos valóricos y sociales. Así, la muestra queda formada por 7 de 8 profesores jefes del primer ciclo básico de la escuela; seis mujeres y un hombre, cuyas edades fluctúan 30 y 55 años.

\subsection{Unidades de estudio}

\subsubsection{Práctica docente:}

Incluye los comportamientos normativos, comportamientos afectivos y la conducción del proceso de enseñanza por parte del docente. 37 Ministerio de Educación de Chile, Bases curriculares Primero a Sexto Básico (Santiago: Unidad de Currículum y
Evaluación, Ministerio de Educación, República de Chile, 2018). 
Comportamiento Normativo del Docente: Entendido como el conjunto de parámetros que el profesor establece para regular la conducta de los alumnos en la sala de clases y en la escuela $^{38}$

Se observa a través de:

- Normas concretas: Identificadas a través de indicaciones o llamadas de atención por incumplimiento.

- Normas abstractas: Identificadas a través de juicios de valor en torno a un valor específico.

- Consistencia de las normas indicadas.

Comportamiento Afectivo del Docente: Entendido como el conjunto de medios a través del cual el profesor presenta o señala una norma concreta o abstracta (Tipo de vehículos) y los gestos de atención y consideración hacia demandas, necesidades, intereses o problemas de los estudiantes (Expresiones afectivas)

- Tipos de vehículos: Se identifican a través del comportamiento verbal y no verbal del profesor al hacer alusiones a las normas y realizar juicios de valor.

- Expresiones afectivas: Se identifican a través del comportamiento verbal y no verbal del profesor al atender las demandas y necesidades de los alumnos.

Conducción de los procesos de enseñanza: Se refiere a la orientación de las actividades de enseñanza. Se observa a través de la promoción de reflexión en las actividades académicas o la existencia de actividades expositivas en el aula.

Para describir las prácticas docentes en el aula de clases, se considera la mayor o menor referencia a la norma realizada por el profesor y el mayor o menor uso de la autoridad para hacer cumplir las normas.

\subsubsection{Interacción entre alumnos:}

Se refiere a aquellas características de la interacción entre alumnos de un mismo curso, según la declaración de padres, profesores y directivos de la escuela. Incluye la

${ }^{38}$ C. Fierro y P. Carbajal, El docente y los valores desde su práctica. Sinéctica 22 (2003): 3 - 11. 
interacción prosocial en la cual una o más personas resultan beneficiadas e interacción violenta, en la cual una o más personas resulta(n) dañada(s).

Interacción prosocial: Comportamientos llevados a cabo voluntariamente para ayudar o beneficiar a otros, tales como compartir, acompañar y ayudar ${ }^{39}$. Son además una de las conductas a través de las cuales se ejerce la responsabilidad social ${ }^{40}$.

Interacción Violenta: Comportamientos llevados a cabo en una situación en que dos o más individuos se encuentran en una confrontación en la cual una o más personas afectadas sale perjudicada, siendo agredida física o psicológicamente ${ }^{41}$. Para este estudio se considerará interacción violenta a las conductas de: Desatención, Desconsideración, Interrupción, Egoísmo y, Agresiones físicas o verbales.

\subsection{Instrumentos}

\subsubsection{Pauta de observación de la práctica docente}

Elaborada para este estudio a partir del modelo de Oferta Valoral Docente de Fierro y Carbajal $^{42}$. Incluye tres dimensiones de la práctica docente:

- Comportamiento normativo de los profesores: normas concretas; normas abstractas; consistencia

- Comportamiento afectivo de los profesores: vehículos utilizados y expresiones afectivas utilizadas.

- Conducción de los procesos de enseñanza identificada en los profesores jefes del primer ciclo básico: descripción de actividades expositivas realizadas y descripción de actividades de reflexión realizadas (académica / valoral).

\footnotetext{
${ }^{39}$ M. Martí, Razonamiento moral y prosocialidad (Madrid: Editorial CCS, 2010).

${ }^{40}$ G. Navarro, Comportamiento socialmente responsable. En Responsabilidad social universitaria, una manera de ser universidad teoría y práctica de la experiencia chilena (Santiago: Proyecto Universidad Construye País, 2006).

41 J. Planella, Repensar la violencia: usos y abusos de la violencia como forma de comunicación en niños y adolescentes en situación de riesgo social. Revista de Intervención Socioeducativa, 10 (1998): 92 - 107.

${ }^{42}$ C. Fierro y P. Carbajal, El docente y los valores desde su práctica. Sinéctica 22 (2003): 3 - 11.
} 


\subsubsection{Pauta de entrevista con lista de chequeo}

Instrumento elaborado para este estudio; contempla 8 comportamientos entre los cuales el entrevistado indica aquellos que ocurren con mayor frecuencia en la interacción entre alumnos: compartir, acompañar, ayudar; desatención al otro, desconsideración, interrupción, egoísmo, agresión física, agresión verbal.

\subsection{Procedimiento}

La recogida de datos se realiza durante los meses de mayo y junio del año 2011. La observación se realiza por una estudiante en Práctica de Psicología en los 7 cursos. Se solicita la autorización al director de la escuela y con ella, se conversa con cada profesor jefe para dar a conocer la metodología de la observación y solicitar consentimiento informado. A los profesores se les indica que la observadora se ubica en el fondo de la sala de clases y que es necesario que no la consideren durante la hora de clase. Paralelamente y durante el mismo período se observa las interacciones entre estudiantes y se entrevista a profesores, apoderados y directivos para caracterizar la interacción entre estudiantes. Resultados de entrevistas y observaciones de las interacciones entre estudiantes se sistematizan en la lista de chequeo descrita en el punto anterior.

\section{Análisis de datos y resultados}

Luego de la observación, los datos son sistematizados en tablas y posteriormente, analizados según el modelo de OVD y en función de los objetivos de la investigación (Tabla 1). 
Tabla 1

Oferta valoral docente de cada profesor

\begin{tabular}{|c|c|c|c|c|c|c|c|c|}
\hline \multicolumn{2}{|c|}{ Componentes OVD } & 1 & 2 & 3 & 4 & 5 & 6 & 7 \\
\hline \multirow[t]{3}{*}{$\begin{array}{l}\text { Comportamiento } \\
\text { Normativo }\end{array}$} & $\begin{array}{l}\text { Normas } \\
\text { Concretas }\end{array}$ & Sí & Sí & Sí & Sí & Sí & Sí & Sí \\
\hline & $\begin{array}{l}\text { Normas } \\
\text { abstractas }\end{array}$ & N.O. & N.O. & Sí & N.O. & N.O. & Sí & Sí \\
\hline & $\begin{array}{l}\text { Consistencia } \\
\text { normativa }\end{array}$ & No & $\mathrm{Si}$ & No & No & No & Sí & No \\
\hline \multirow[t]{2}{*}{$\begin{array}{l}\text { Comportamiento } \\
\text { Afectivo }\end{array}$} & $\begin{array}{l}\text { Principal } \\
\text { tipo de } \\
\text { vehículo } \\
\text { utilizado }\end{array}$ & Tipo 2 & Tipo 2 & Tipo 2 & Tipo 2 y 3 & Tipo 2 & Tipo 1 & Tipo 2 y 3 \\
\hline & $\begin{array}{l}\text { Principal } \\
\text { grupo de } \\
\text { expresiones } \\
\text { afectivas } \\
\text { utilizadas }\end{array}$ & Grupo 2 & Grupo 2 & Grupo 3 & Grupo 3 & Grupo 3 & Grupo 2 & Grupo 2 \\
\hline \multirow[t]{2}{*}{$\begin{array}{l}\text { Conducción } \\
\text { Procesos de } \\
\text { Enseñanza }\end{array}$} & $\begin{array}{l}\text { Actividad } \\
\text { Expositiva- } \\
\text { Actividad } \\
\text { Reflexiva }\end{array}$ & $\begin{array}{l}\text { Actividad } \\
\text { Expositiva }\end{array}$ & $\begin{array}{l}\text { Actividad } \\
\text { Reflexiva }\end{array}$ & $\begin{array}{l}\text { Actividad } \\
\text { Expositiva }\end{array}$ & $\begin{array}{l}\text { Actividad } \\
\text { Expositiva }\end{array}$ & $\begin{array}{l}\text { Actividad } \\
\text { Expositiva }\end{array}$ & $\begin{array}{l}\text { Actividad } \\
\text { Reflexiva }\end{array}$ & $\begin{array}{l}\text { Actividad } \\
\text { Expositiva }\end{array}$ \\
\hline & $\begin{array}{l}\text { Reflexión } \\
\text { Académica/ } \\
\text { Valoral }\end{array}$ & & Académica & & & & $\begin{array}{l}\text { Académica/ } \\
\text { Valoral }\end{array}$ & \\
\hline
\end{tabular}

**N.O.: No observado

1.En relación a las Prácticas Docentes, los resultados muestran lo siguiente:

a). Comportamiento normativo del docente: el 100\% de los profesores usan normas concretas, por ejemplo "no faltar el respeto", "escuchar a la profesora”; el 43\% usa además, normas abstractas tales como "respeto", "solidaridad"; mientras que sólo el 28\% presenta consistencia normativa. 
O人лмв Lu Wimblu, Rev. Estud. de Psicología UCR, 15(1) 2020 (Enero-Junio): 7-34 /ISSN: 1659-2107

b). Comportamiento afectivo del docente: el vehículos más usado es de tipo 2 (86\% de los profesores), es decir, vehículos que hacen alusiones puntuales a la norma incumplida o el comportamiento que se debía observar; carecen de explicaciones que aluden al sentido de las normas o la importancia de cumplirlas y centra el contacto con las normas en la referencia a las mismas, sin aportar los elementos de juicio necesario para su comprensión, por ello limita las oportunidades de interiorización de las normas concretas, y el desarrollo de la autonomía en los alumnos. El 29\% de los profesores usa vehículos de tipo 3, es decir, aquellos que se basan en una actuación discrecional del docente para hacer cumplir las normas, desplazando la atención desde la norma hacia su persona como motivo para cumplirla, y no al sentido y los valores que están detrás de ellas. Al colocar al docente como referencia para el cumplimiento de las normas, se erige como eje central de donde parten las razones para su cumplimiento. Por lo tanto, propicia un escaso razonamiento sobre el sentido de las normas, en consecuencia, no promueve las oportunidades de toma de rol. El 14\% de los profesores utiliza vehículos de tipo 1, es decir, aquellos en que se invita al niño a ponerse en el lugar de la otra persona. Sirve para ampliar su perspectiva social, es decir, ayuda al alumno a tomar en cuenta el punto de vista del otro y el daño que puede causar una acción a otra persona. Este tipo de vehículos tiene repercusiones positivas en el desarrollo de la moralidad, generando oportunidades para la toma de rol y por lo tanto, para la interiorización de las normas concretas. Dentro de los tres tipos identificados, los vehículos específicos más observados son la felicitación $(100 \%$ de los profesores observados); expresar disgusto personal por la falta (86\%); recibir la acusación (86\%) y gritar al alumno (71\%). No se observó vehículos tipo 4, es decir, aquellos en que se utiliza la fuerza de la autoridad como recurso habitual para hacer cumplir las normas.

Las expresiones afectivas más usadas son del grupo 2 (observadas en el $57 \%$ de los profesores), es decir, aquellas que se centran en atender de manera puntual una demanda de alumnos o del grupo (atención a apoyar y corregir errores puntuales de aquellos, dirigir actividades, invitar a participar a través de gestos, etc.) y del grupo 3 (observadas en el $43 \%$ de los profesores), que son expresiones afectivas que aluden a la persona del docente o a su actuación discrecional como vía para atender las demandas de los alumnos, en este caso las diferencias de contenido. Este grupo genera la experiencia de incertidumbre en los alumnos, ya que el trato de hoy no es garantía de que mañana sea el mismo, el que lo sea 
O人лмв Lu Wimblu, Rev. Estud. de Psicología UCR, 15(1) 2020 (Enero-Junio): 7-34 /ISSN: 1659-2107

depende de la figura del docente. Al interior de estos grupos, las expresiones específicas más usadas son apoyar o ayudar (71\%), expresar disgusto o reprobación (71\%) y abrazar o acariciar (57\%). No se observó expresiones afectivas del grupo 1, que manifiestan atención al alumno, reconocimiento de sus necesidades y logros y promueven distensión en el grupo ni del grupo 4, basadas en la imposición o el uso de la fuerza de la autoridad en relación con las demandas de los alumnos.

c). Conducción de los procesos de enseñanza: el $71 \%$ de los profesores observados orienta las actividades de enseñanza hacia la exposición/mecanicista y un 29\% promueve la reflexión académica. Solo 1 de 7 profesores promueve la reflexión valoral.

2.Con relación a la interacción entre alumnos, los resultados muestran lo siguiente:

a) Lo declarado en entrevistas se puede sintetizar en que padres, profesores y directivos clasifican como interacción violenta el comportamiento de los alumnos en el establecimiento educacional.

b) Mediante lista de chequeo, se puede establecer que en $29 \%$ de los cursos (2 de 7), las interacciones más frecuentes en los niños son violentas, prevaleciendo en ellas las siguientes conductas: Interrupción, Agresiones físicas o verbales, Desatención, Desconsideración y Egoísmo. Solo en 2 de siete cursos se dan con mayor frecuencia las interacciones prosociales, caracterizadas por conductas tales como Compartir, Acompañar y Ayudar.

c) La interacción de los alumnos de cada curso se caracteriza principalmente por las conductas identificadas en la tabla 2. 
Tabla 2

Descripción de interacción de los alumnos de cada curso.

\begin{tabular}{|l|l|l|l|l|l|l|}
\hline Curso 1 & Curso 2 & Curso 3 & Curso 4 & Curso 5 & Curso 6 & Curso 7 \\
\hline Interrupción & Interrupción & Desatención & Desatención & Desatención & Interrupción & Agresiones \\
Agresiones & Compartir & Desconsider & Desconsider & Agresiones & Desatención & físicas \\
Verbales & Acompañar & ación & ación & verbales & Compartir & Desconsider \\
Consideraci & Ayudar & Compartir & Agresiones & Desconsider & Ayudar & ación \\
ón & & Ayudar & (f y v) & ación & Acompañar & Agresiones \\
Ayuda & & Acompañar & Interrupción & Ayuda & & verbales \\
Compartir & & & Acompañar & Acompañar & & Ayudar \\
\hline
\end{tabular}

\section{Discusión}

La Oferta Valoral Docente (OFV) es todo aquello que el profesor transmite en términos de valores y las oportunidades que ofrece para el desarrollo de la moralidad de sus alumnos $^{43}$. Son las practicas docentes uno de los factores que influyen en la aparición de conductas prosociales, las cuales a su vez dependen también del desarrollo moral que van adquiriendo los alumnos ${ }^{44}$.

En el caso de los 7 profesores jefes observados en la Escuela, es posible identificar en todas las aulas normas concretas que están claras. Esto afecta la socialización del alumnado, pudiendo conocer aquellos comportamientos permitidos y aquellos prohibidos dentro de la sala de clases. Sin embargo, y así como se identificó en el estudio de Fierro y Carbajal $^{45}$ donde un $86 \%$ de las alusiones a normas concretas fueron por llamadas de atención y un $14 \%$ por indicaciones, en las salas de clases observadas ocurre lo mismo, predominando un comportamiento reactivo frente a las distintas situaciones en el aula, dificultando el aprendizaje del cómo comportarse y reforzando el cómo no hacerlo.

\footnotetext{
${ }^{43}$ C. Fierro y P. Carbajal, El docente y los valores desde su práctica. Sinéctica 22 (2003): 3 - 11.

${ }^{44}$ M. Martí,. Razonamiento moral y prosocialidad (Madrid: Editorial CCS, 2010).

${ }^{45}$ C. Fierro y P. Carbajal, El docente y los valores desde su práctica. Sinéctica 22 (2003): 3 - 11.
} 
Con relación a las normas abstractas solo se identificaron en las prácticas docentes de tres de los siete profesores observados. Se identificaron juicios de valor, indicándoles a los alumnos qué es lo esperado por sobre aquello que no deben realizar, sin embargo, aquellos juicios no fueron argumentados de forma clara, sino que más bien, entregados sin mayor reflexión; esto facilita la socialización de las normas, pero dificulta su interiorización. La baja aparición de juicios de valor durante las observaciones se relaciona también con lo observado por Fierro y Carbajal ${ }^{46}$ quienes encontraron que, de las normas declaradas, un $94 \%$ corresponde a normas concretas y solo un $6 \%$ a valores universales o normas abstractas.

En cuanto a la consistencia normativa, solo dos de los siete profesores fueron consistentes, es decir promovieron la estabilidad y predicción en las normas declaradas. Esto se relaciona directamente con el comportamiento de sus cursos, los cuales presentan la mejor convivencia dentro de los siete cursos observados.

La flexibilidad en las normas declaradas dificulta enormemente el desarrollo hacia la autonomía moral $^{47}$, ya que aumentan el grado de dependencia de la actividad de la figura de autoridad para su cumplimiento, en lugar del aprendizaje de ellas. Esto conduce a que los alumnos las respeten sólo en presencia de aquella figura y en su ausencia, tanto en la sala de clases como en recreo, no se comporten de acuerdo a lo normado.

El comportamiento afectivo observado en los docentes, en seis de las siete prácticas observadas, se compone principalmente de vehículos del tipo dos, los cuales se caracterizan por alusiones puntuales a la norma incumplida o al comportamiento que se debía observar. Estos vehículos carecen de explicaciones que aluden al sentido de las normas o la importancia de cumplirlas. En virtud de lo anterior el contacto con las normas se centra en la referencia a las mismas, sin aportar los elementos de juicio necesario para su comprensión y generación de oportunidades de interiorización de las normas concretas, y el desarrollo de la autonomía en los alumnos ${ }^{48}$. Esto contribuye a entender el comportamiento característico de los alumnos de la escuela, donde el primer ciclo básico en su generalidad

\footnotetext{
${ }^{46}$ C. Fierro y P. Carbajal, El docente y los valores desde su práctica. Sinéctica 22 (2003): 3 - 11.

${ }^{47}$ C. Fierro y P. Carbajal, El docente y los valores desde su práctica. Sinéctica 22 (2003): 3 - 11.

${ }^{48}$ C. Fierro y P. Carbajal, El docente y los valores desde su práctica. Sinéctica 22 (2003): 3 - 11.
} 
logra comportarse adecuadamente en presencia de la figura de autoridad, pero no logra mantener aquellas conductas en su ausencia. Además, dos de los seis profesores que utilizan principalmente vehículos del tipo 2, utilizan también en la misma proporción vehículos de tipo 3, los cuales se relacionan directamente con la figura del profesor, es decir, se basan en su actuación discrecional y establecimiento de las normas como un asunto referido a él mismo y sus preferencias. Así el profesor se coloca como razón para hacer cumplir las normas, desplazando la atención de los alumnos hacia su persona y no al sentido y los valores que están detrás de ellas. El profesor se erige como eje central de donde parten las razones para su cumplimiento. En estos dos casos el desarrollo hacia la autonomía moral y por lo tanto, la aparición de conductas prosociales, se ve aún más dificultado ${ }^{49}$.

Se observa solo en una profesora la utilización principalmente de vehículos tipo 1, los cuales promueven la toma de rol, ayudando al alumno a considerar las experiencias e ideas de otros, pudiendo comprender el daño o beneficio que puede causar con su actuar. Este tipo de vehículo tiene repercusión positiva en el desarrollo de la moralidad, generando oportunidades para la toma de rol y, por lo tanto, para la interiorización de las normas concretas y facilitación del desarrollo hacia la autonomía moral. El grupo curso que atiende esta profesora es también aquel que mantiene por mayor tiempo la atención en las tareas indicadas por la docente y aquel donde la convivencia y el volumen de ruido de la sala de clases fue más adecuado para generar instancias efectivas de enseñanza-aprendizaje.

En cuanto a las expresiones afectivas, se identifica que cuatro de los siete docentes observados utilizan el grupo 2, es decir, se centran en atender de manera puntual las demandas de alumnos o del grupo, sin generar mayores instancias de interacción y por lo tanto sin atender a uno de los principales objetivos de la educación chilena, aprender a convivir. Se identifica que en la mayoría de las salas de clases los alumnos manifiestan sus necesidades individuales, las cuales efectivamente son atendidas por el docente a cargo, sin embargo, no implican la participación de los compañeros presentes en su satisfacción, dificultando así la toma de rol y por lo tanto la interiorización y posterior autonomía moral.

\footnotetext{
${ }^{49}$ C. Fierro y P. Carbajal, El docente y los valores desde su práctica. Sinéctica 22 (2003): 3 - 11.
} 


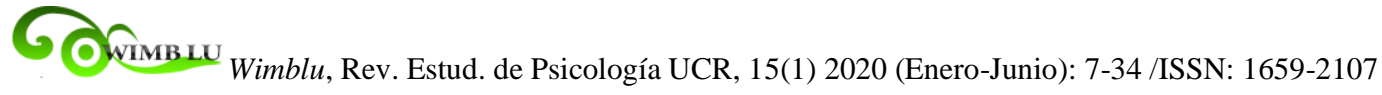

Los otros tres docentes utilizan expresiones afectivas del grupo 3, las cuales implican una alta alusión a la autoridad como recurso para sustentar un juicio, con una muy baja elaboración sobre las razones que sustentan una afirmación determinada. La figura del docente y sus creencias son la fuente de la cual emana la autoridad para sustentar la validez de un juicio de valor. Su formulación misma invita a la adhesión, no a la reflexión en torno a su contenido sin otorgar oportunidades para que los alumnos elaboren sus propios razonamientos, limitando el desarrollo de su moralidad y en el caso concreto de esta escuela promoviendo el comportamiento adecuado solo en presencia de la figura de autoridad.

En cuanto a la conducción de procesos de enseñanza, solo 2 de los siete profesores realizan actividades reflexivas, las que son principalmente de contenido académico. Sin embargo, se observa que en aquellos cursos donde los alumnos son desafiados cognitivamente la atención e interés son mantenidos por mayor tiempo en las tareas académicas, facilitando el proceso de enseñanza-aprendizaje.

Los otros 5 docentes utilizan actividades que son exclusivamente mecánicas y expositivas, perdiendo rápidamente el interés de los alumnos y dificultando la posibilidad de analizar situaciones de la vida diaria o dilemas morales elaborando razonamientos propios sobre ellos.

La baja aparición de la reflexión registrada en este establecimiento se compara con lo observado por Fierro y Carbajal $^{50}$, quienes identificaron que un $95 \%$ de las actividades realizadas en el aula corresponde a actividades elementales o mecánicas y solo un $5 \%$ a actividades de mayor complejidad como secuencias de reflexión.

Al comparar la convivencia escolar entre los diferentes cursos, se identifica que es la consistencia en el comportamiento normativo el factor común entre los cursos con mejor comportamiento. Dos de los siete profesores fueron consistente con lo declarado y fueron esos dos cursos los que presentaros mayor atención e interés en las actividades académicas realizadas. Además, ambos profesores utilizan actividades reflexivas, sin embargo, sólo uno de ellos las utiliza en contenidos académicos y valores, promoviendo aún más el desarrollo hacia una autonomía moral.

\footnotetext{
${ }^{50}$ C. Fierro y P. Carbajal, El docente y los valores desde su práctica. Sinéctica 22 (2003): 3 - 11.
} 
O人лмв Lu Wimblu, Rev. Estud. de Psicología UCR, 15(1) 2020 (Enero-Junio): 7-34 /ISSN: 1659-2107

Al describir y analizar las prácticas docentes de los profesores del primer ciclo básico, se comprende cómo aquellas dificultan la aparición de conductas prosociales. Las conductas prosociales podrán actuar como factor protector frente a la conducta violenta, la cual es indicada como uno de los principales problemas en el establecimiento.

La oferta valoral docente es muy disminuida y no se ofrecen oportunidades efectivas para el desarrollo de la moralidad de los alumnos. Por el contrario, las prácticas de los profesores están dificultando el desarrollo hacia la autonomía moral.

Se puede identificar una alta socialización de las normas, lo cual se observa en el comportamiento en el aula y fuera de ella, donde los alumnos en su mayoría cambian su comportamiento frente a la presencia de sus profesores jefes. Sin embargo, como las prácticas de los profesores promueven la socialización y no la interiorización y posterior autonomía, hay esfuerzos de éstos que se pierden durante la jornada escolar. Así, un profesor pasa la mayor parte del día, por ejemplo, indicando cómo no comportarse, llamando la atención o aumentando el volumen de voz para ser escuchado. Pero a su vez refleja inconsistencia normativa, utiliza vehículos del tipo 2 ó 3 y no promueve actividades reflexivas. Por lo tanto, las prácticas utilizadas no son coherentes con los objetivos que se propone, volviéndose inefectivas.

\section{Conclusiones}

La aparición de conductas prosociales en los alumnos del establecimiento se ve obstruida principalmente por la inconsistencia en las normas y por el énfasis que tiene en su cumplimiento la figura de autoridad que es el profesor jefe, observándose comportamientos contradictorios a lo normado en el aula de clases y fuera de ella.

Es importante destacar que las prácticas docentes observadas no generan la conducta violenta, sino que no están promoviendo el desarrollo moral de los alumnos y por lo tanto, no facilitan la aparición de conductas prosociales, las cuales funcionan como factor protector ante esta conducta problema. 
6 Oิлив Lu Wimblu, Rev. Estud. de Psicología UCR, 15(1) 2020 (Enero-Junio): 7-34 /ISSN: 1659-2107

En un contexto altamente vulnerable como el de este establecimiento, la figura del profesor se vuelve trascendental, ya que, al lograr consistencia en su propio comportamiento y coherencia entre todo el cuerpo docente, sus prácticas se vuelven efectivas en cuanto al desarrollo de la moralidad y por lo tanto, oportunidades de aprendizaje significativo. Por lo tanto, es de importancia guiar al cuerpo docente hacia la consistencia normativa, uso de vehículos tipo 1 y promoción de actividades reflexivas.

Estudios en esta área pueden ser significativos, principalmente al generar modelos de entrenamiento con profesores que permitan que sus prácticas en el aula y fuera de ella se vuelvan efectivas en esta dirección, promoviéndose el desarrollo moral, la aparición de conductas prosociales y disminuyendo la frecuencia de conductas violentas.

\section{Referencias bibliográficas}

Alonso, S., Cáceres, M. e Hinojo, M. La Convivencia Escolar y el Conocimiento de un Grupo Mediante la Sociometría: Entrenamiento en Habilidades Sociales. Revista Iberoamericana sobre Calidad, Eficacia y Cambio en Educación 8 (2010): 45-57.

Cangas, Vázquez, Pérez-Fuentes, Padilla y Miras, Evaluación de la violencia escolar y su afectación personal en una muestra de estudiantes europeos. Psicothema 19, (2007): 114 -119 .

De la Villa, M., García, A., Cuetos, G. y Sirvent, C., Violencia en el noviazgo, dependencia emocional y autoestima en adolescentes y jóvenes españoles. Revista Iberoamericana de Psicología y Salud 8, no. 2 (2017): 96-107.

Fierro, C. y Carbajal, P., El docente y los valores desde su práctica. Sinéctica 22, (2003): 3 -11 .

Fierro, C. y Carbajal, P., Mirar la práctica docente desde los valores. Ciudad de México: Gedisa, 2003.

Fuentes, J.L., Evolución y perspectivas de la educación moral. Revista española de pedagogía REP 75, (2018).

Hersh, R., Reimer, J., y Paolitto, D., Crecimiento Moral de Piaget a Kohlberg. Madrid: Narcea, 2002. 
Ianni, N., La convivencia escolar: una tarea necesaria, posible y compleja. Monografías virtuales: Ciudadanía, democracia y valores en sociedades plurales 2, (2003). Extraído de http://www.oei.es/valores2/monografias/monografia02/reflexion02.htm

Inglés, C., Martínez-González, E. y García-Fernández, J., Conducta prosocial y estrategias de aprendizaje en una muestra de estudiantes españoles de Educación Secundaria Obligatoria. European Journal of Education and Psychology. 6, no. 1 (2013): 33-53.

Macías, D., Santamaría, A., Zavala, A., Adoví, M. y Pincay, J., Gestión pedagógica: clima social en el aula, desde la percepción de estudiantes y profesores. Revista electrónica Formación y Calidad Educativa REFCalE 4, no. 1 (2016).

Martí, M., Razonamietno moral y prosocialidad. Madrid: Rditorial CCS, 2010.

Mertz, C., La prevención de la violencia en las escuelas. Programa Paz Educa, 2006.

Ministerio de Educación de Chile, Bases curriculares Primero a Sexto Básico (Santiago: Unidad de Currículum y Evaluación, Ministerio de Educación, República de Chile, 2018).

Navarro, G., Vaccarezza, G., González, MG y Catalán, R. Construcción de conocimiento en educación superior; educación de competencias genéricas en la Universidad de Concepción. Concepción: Editorial Universidad de Concepción, 2015.

Pascual, A., Implicaciones psicopedagógicas de un desarrollo moral íntegro: La educación holística, Universidad de Zaragoza. Zaragoza: Universidad de Zaragoza, 1999.

Planella, J., Repensar la violencia: usos y abusos de la violencia como forma de comunicación en niños y adolescentes en situación de riesgo social. Revista de Intervención Socioeducativa 10 (1998): 92 - 107.

Quintero, J., Estrategias docentes como práctica de la teoría pedagógica. CONHISREMI, Revista Universitaria de Investigación y Diálogo Académico 4 (2008).

Renes P. y Caldeiro MC., Nuevos escenarios sobre la educación en valores en las escuelas. Inovare 04 2, (2017).

Ruiz, J., Ponce de León, A., Sanz, E. y Valdemoros, M., La educación en valores desde el deporte: investigación sobre la aplicación de un programa integral en deportes de equipo RETOS. Nuevas Tendencias en Educación Física, Deporte y Recreación, no. 28 (2015): 270-275 
O.

Velázquez, M.G. y Candela, A., Educación, valores y desarrollo moral. Formación valoral y ciudadana, Gernika 2 (2006): 335-356

Zabalza, M., Situación de la convivencia escolar en España: Políticas de intervención. Revista Interuniversitaria de Formación de Profesorado 44 (2002): 139 - 174. 